\title{
BMJ Open Mental health in the second year of the COVID-19 pandemic: protocol for a nationally representative multilevel survey in Serbia
}

\author{
Nadja P Marić, ${ }^{1}$ Ljiljana B Lazarević, ${ }^{2}$ Ljiljana Mihić, ${ }^{3}$ Milica Pejovic Milovancevic, ${ }^{4}$ \\ Zorica Terzić, ${ }^{1}$ Oliver Tošković, ${ }^{5}$ Jovana Todorović, ${ }^{1}$ Olivera Vuković, ${ }^{1}$ \\ Goran Knezevic (i) ${ }^{5}$
}

To cite: Marić NP, Lazarević LB,

Mihić L, et al. Mental

health in the second year of the COVID-19 pandemic: protocol for a nationally representative multilevel survey in Serbia. BMJ Open 2021;11:e053835. doi:10.1136/ bmjopen-2021-053835

- Prepublication history for this paper is available online. To view these files, please visit the journal online (http://dx.doi. org/10.1136/bmjopen-2021 053835).

Received 26 May 2021 Accepted 09 September 2021

Check for updates

(C) Author(s) (or their employer(s)) 2021. Re-use permitted under CC BY-NC. No commercial re-use. See rights and permissions. Published by BMJ.

${ }^{1}$ Univerzitet u Beogradu Medicinski fakultet, Beograd, Serbia

${ }^{2}$ Institute of Psychology, Univerzitet u Beogradu Filozofski Fakultet, Belgrade, Serbia ${ }^{3}$ Department of Psychology, Univerzitet u Novom Sadu Filozofski fakultet, Novi Sad, Vojvodina, Serbia

${ }^{4}$ Department of Psychiatry, Univerzitet u Beogradu Medicinski fakultet, Beograd, Serbia

${ }^{5}$ Department of Psychology, Univerzitet u Beogradu Filozofski Fakultet, Belgrade, Serbia

Correspondence to Professor Goran Knezevic; gknezevi@f.bg.ac.rs

\section{ABSTRACT}

Introduction The COVID-19 pandemic is likely to have a prolonged impact on mental health $(\mathrm{MH})$; however, the long-term MH effects of the COVID-19 pandemic remain unknown. The Serbian national survey-CoV2Soul. RS - was launched to document the MH status of the Serbian population following the COVID-19 pandemic and to contribute to an international evidence base about $\mathrm{MH}$ prevalence rates during different phases of the pandemic. Methods and analysis This cross-sectional study was designed to collect a nationally representative sample ( $N=1200$; age 18-65 years; estimated start/end-June/ November 2021) using multistage probabilistic household sampling. Trained staff will conduct in-person diagnostic interviews. A battery of self-report instruments will be administered to assess the quality of life (QoL), general distress and associated protective and harmful psychological and societal factors. Analyses will be conducted to delineate the prevalence rates of $\mathrm{MH}$ disorders, how MH conditions and QoL vary with respect to sociodemographic variables, personality, health status and traumatic events during the COVID-19 pandemic, and to test how these relations depend on geographical region. Moreover, this study was designed to explore mechanisms linking personality and the perception of pandemic consequences and associated distress. Prevalence rates of $\mathrm{MH}$ disorders will be calculated using descriptive statistics. For additional analyses, we will use correlations, analysis of variance and regression analyses. The hierarchical structure of the data will be explored using multilevel random coefficient modelling. Structural equation modelling will be used to investigate the indirect effects of personality on distress through relevant variables. Ethics and dissemination Ethical Committees of the Faculty of Medicine (1322-VII/31) and Faculty of Philosophy in Belgrade (02-33/273) and Faculty of Philosophy in Novi Sad (05-27, br.893/1) approved the protocol. Only respondents able to provide informed consent will participate in the study. Research reports will be submitted to peer-reviewed journals and the results will be placed on the website www.cov2soul.rs to be available to funders, researchers, policy-makers and interested laypeople, and will be advertised through social media. Trial registration number NCT04896983.
Strengths and limitations of this study

- CoV2Soul.RS will enable the assessment of mental health $(\mathrm{MH})$ disorder prevalence in a representative national adult population in relation to COVID-19.

- Due to the prolonged duration of the COVID-19 pandemic, a more adequate evaluation of the full extent of COVID-related MH conditions is warranted.

- $\mathrm{MH}$ disorders will be assessed in person, using a structured diagnostic tool administered by professionals comprehensively trained and supervised to collect data by multistage probabilistic household sampling

- The scientific community will benefit from an opportunity to include obtained data in forthcoming international meta-analytical studies.

- The primary limitation is the lack of reliable baseline national statistics which limits the possibility to analyse trends in the prevalence of mental disorders prior to and during the COVID-19 pandemic.

\section{INTRODUCTION}

Viral outbreaks, such as the COVID-19 pandemic, are associated with short-term and long-term psychological and societal distress. ${ }^{1}$ For instance, the first massive viral infection outbreak in the 21st century-SARS (in 2003), resulted in a substantial increase in mental health (MH) symptoms. ${ }^{2}$ Alternatively, the prolonged stress associated with living through a pandemic may promote resiliency, as some people can find strengths that lead towards post-traumatic personal growth. $^{3}$ The COVID-19 pandemic has resulted in unprecedented societal burden, from a burden on health systems, inevitable deterioration of the economy, higher rates of unemployment, global social restrictions, lockdowns and disturbing news stories that impose considerable stress. ${ }^{4-6}$ These factors could trigger new-onset of $\mathrm{MH}$ disorders or worsen previously developed MH disorders. ${ }^{7}$ 
In the UK Household Longitudinal Study conducted in 2018/2019 and immediately after the pandemic outbreak (2020), a screening instrument showed that the prevalence of clinically significant levels of $\mathrm{MH}$ disorders increased from approximately $19 \%-27 \% .^{8}$ In the Czech Study which explored a nationally representative sample at two assessment points (2017 and 2020), the baseline measurements showed the presence of at least one $\mathrm{MH}$ disorder in $20 \%$ of the sample, whereas the prevalence of at least one $\mathrm{MH}$ disorder was $30 \%$ within a few months after the COVID-19 outbreak. ${ }^{7}$

The majority of the published studies have been conducted within the first months of the pandemic, not taking into account the progression (or regression) of MH symptoms throughout the pandemic. Further, most studies relied on diagnostic screening tools or diagnostic questionnaires with a short reference period. ${ }^{9}{ }^{10}$ Indeed, most studies conducted during the COVID-19 pandemic have relied on self-report surveys using convenience sampling and abbreviated measurement strategies. ${ }^{11}$ Due to the over-reliance on self-report surveys, the overall impact of COVID-19 on MH is taking months to become fully apparent in the general population.

MH status of the population is a multifactorial complex phenomenon, depending on many factors including proximity to the virus (history of CoV2-infection and related exposures), general health status, individual psychological dispositions, traumatic events during pandemics and multifaceted contextual societal factors (SFs). Therefore, conducting a community-based survey to assess the prevalence of psychiatric disorders and to elucidate the key mechanisms of poor MH using multilevel analyses (multilevel random coefficient modelling $(\mathrm{MRCM})^{12}$ is vital for preparing to provide accessible and timely pandemic and postpandemic services. Psychiatrists should alert policy-makers and other authorities of the long-term consequences and expected changes in $\mathrm{MH}$ service demand. ${ }^{13}$ When the pandemic is over, it might be that MH services face a shortage of professionals due to burnout and mental exhaustion. ${ }^{14}$

Until now, only one study of a nationally representative sample used an established psychodiagnostic instrument to detect $\mathrm{MH}$ disorders following the COVID-19 pandemic. ${ }^{7}$ However, this study was conducted close to the onset of the COVID-19 pandemic in the Czech Republic, so the prolonged $\mathrm{MH}$ outcomes of the pandemic remain unclear. To the best of our knowledge, nationally representative field research using structured diagnostic interviews to explore $\mathrm{MH}$ status and other $\mathrm{MH}$ indices (quality of life (QoL), level of distress) after prolonged exposure to pandemic has not been established. Moreover, no one explored all aforementioned factors in relation to, (level of distress) after prolonged exposure to pandemic has not been established. Moreover, no one explored all aforementioned factors in relation to (level of distress) after prolonged exposure to pandemic has not been established. Moreover, no one explored all aforementioned factors in relation to SFs specific to the local municipalities.
The current study was designed to address these gaps in knowledge by: (1) Assessing prevalence rates of $\mathrm{MH}$ disorders and associated QoL in a nationally representative Serbian sample, and identifying factors (including sociodemographic data, history of Cov2-infection, Cov-2 distress and general health status) related to the occurrence of these disorders; (2) Exploring, using multilevel modelling, how MH conditions and QoL vary with respect to sociodemographic variables, personality, health status and traumatic events during pandemics, and to find how these relationships depend on SFs characterising municipalities in which respondents live and (3) Exploring the perception of pandemic consequences and associated distress in relation to personality, taking into account possible mediators: feelings of loneliness, religious beliefs and attitude towards the COVID-19 conspiracy theories.

\section{METHODS AND ANALYSIS}

A cross-sectional observational study will include 1200 randomly selected adults aged between 18 and 65 years. Exclusion criteria will be non-fluent Serbian speakers, severe neurological and cognitive impairment leading to an inability to understand items, and hearing-impaired individuals with whom research assistants (RAs) cannot communicate verbally.

\section{Setting and participants}

The sample will be collected via multistage probabilistic household sampling from June to November 2021. The first stage will include a random sampling of municipalities as clusters, and the second stage will include a random sampling of local communities in each municipality. Municipalities and local communities will be sampled from four regions (Capital Belgrade and suburbs, Vojvodina, West and Southeast Serbia) based on the random selection from the database created by the Statistical Office of the Republic of Serbia (including information on the name of the settlement, municipality, region and the number of inhabitants). The third, final stage, will involve the selection of the respondents using a random walk technique performed by RAs. RAs will employ faceto-face interviewing and computer-assisted or paper-andpencil method to administer questionnaires. The number of respondents in each start point will not exceed 13, to guarantee that the principle of randomness will not be jeopardised. The number of respondents will be proportionate to the size of the community, proportionally to the total sample size. Additionally, the minimum number of respondents sampled from the municipality is 18 (taking into account the municipality size proportionally to the sample size) to allow MRCM.

The sample will include respondents from 137 local communities deployed in 63 municipalities. The number of respondents in local communities ranges between 5 and 13 , while at the municipality level it ranges between 18 and 39. A review of the simulation studies showed that higher levels of power were achieved with larger samples 
at level 2 than at level 1 , and suggested the $30 / 30$ rule of thumb (a minimum of 30 groups with 30 individuals in those groups. ${ }^{15}$ Hox $^{16}$ proposed the $50 / 20$ rule (ie, 50 groups with 20 individuals per group). Thus, 63 municipalities (level 2) with 20 individuals per group (level 1) guarantee adequate power to detect fixed effects, variance components and cross-level interactions in multilevel modelling.

In total, our sample will comprise 1200 respondents. This sample size enables detecting the correlation of 0.08 , with a power of 0.80 , at a .05 alpha level. It means that if only $1 \%$ of the variance is shared between $\mathrm{MH}$ variables and COVID-19 exposure indices, the design of our study enables detecting it. Finally, our sample size enables detecting the differences in MH status (any mental disorder, mood disorders, anxiety disorders, suicidality) between those with history of CoV2-infection (or showing COVID-19 distress) of the size that was reported by the recent Czech study ${ }^{7}$ with the power of 0.80 or higher, at the 0.05 alpha level ( $G^{*}$ power). ${ }^{17}$

\section{Procedures}

Data will be collected using a standardised case report form. All instruments except for M.I.N.I. V.7.0.2. Standard ${ }^{18}$ will be collected using an online platform.

\section{Training of RAs}

All RAs will be provided with a comprehensive education to conduct diagnostic interviews and administer the test battery. RAs will be medical doctors and psychologists and will approach potential respondents at their homes. Those willing to participate will give written informed consent. If a respondent is visually impaired or has difficulties reading, RAs will read the items and record the responses of the respondent.

\section{Instruments and measures}

Sociodemographic variables include information on age, gender, education, occupation, income, marital status, household size, religiosity and vaccination status.

The socioeconomic status (SES) will be measured via the social ladder technique. ${ }^{19}$

Health status variables assess the overall health status, including somatic and pre-existing psychiatric conditions. The use of anxiolytics and hypnotics will be explored focusing on the period before and during the pandemic, and 7 days preceding the assessment.

\section{Prevalence of MH disorders}

(A) Structured interview to assess the prevalence of 16 most common MH disorders (M.I.N.I. V.7.0.2-Standard Adult version). To find the prevalence of serious mental disorders (SMI-major depressive disorder, bipolar disorder or any psychotic disorder) we will focus on the current and past depressive, manic/hypomanic or psychotic episodes. For post-traumatic stress disorder, obsessive-compulsive disorder, social phobia, panic disorder and suicidality we will focus on the last month; for eating disorders on the past 3 months, for generalised anxiety disorder and agoraphobia on the past 6 months, while for alcohol and substance use disorder the focus will be on the past 12 months. Antisocial personality disorder prevalence is based on the lifetime perspective.

In addition, the following diagnostic groups will be used (in line with the contemporary classifications of psychiatric disorders): (1) mood disorders (major depressive episode, manic episode and hypomanic episode); (2) psychotic disorders; (3) anxiety disorders (panic disorder, agoraphobia, social phobia and generalised anxiety disorder); (4) obsessive-compulsive disorder; (5) trauma-related disorders (post-traumatic stress disorder); (6) eating disorders and (7) substance-related and addictive disorders.

\section{MH symptom severity}

The suicidality score will be calculated as the sum of the six M.I.N.I. items: wish for death (weight of 1), wish for self-harm (weight of 2), suicidal thoughts (weight of 6), suicide plan (weight of 10), suicide attempt in the past 1 month (weight of 10) and lifetime suicide attempt (weight of 4) (explained in more details by Park et al) ${ }^{20}$ The severity of alcohol and substance use disorder in the past 12 months will be generated by M.I.N.I (mildmoderate-severe). The Patient Health Questionnaire-9 $(\mathrm{PHQ}-9)^{21}$ and the General Anxiety Disorder-7 (GAD$7)^{22}$ - depressive and anxiety symptom severity-will be measured with two aforementioned scales, to conclude about the symptom severity and the current level of distress in all participants. The results will be interpreted as follows: PHQ-9 score 10-14 indicates moderate depression, 15-19 moderate-severe depression and 20 and above severe depressive symptoms, while GAD-7 score of 10 or more is a cut-point for moderate intensity, while 15 or more is representing severe levels of anxiety.

QoL will be assessed using the Manchester Short Assessment of Quality of Life ${ }^{23}$ and a 12-item Short-Form Health Survey. ${ }^{24}$ QoL assumes focusing on satisfaction with life as a whole and with life domains and more precise screening of health status (mental and physical).

\section{Pandemic-related variables}

Cov2-exposure consists of the history of Cov2-infection and other Cov2-related situational risks. History of Cov2infection will be classified as none, mild-moderate (infection without Intensive Care Unit (ICU) admission) and severe (with ICU admission). To assess Cov-2-related situational risks, we will use nine items designed for this study which measure if family members were diagnosed with COVID-19 if someone from the respondent's family and/ or close others died due to COVID-19, did respondent have protective equipment at work, etc.

List of threatening experience ${ }^{25}$ assesses the occurrence of negative life events via 12 items. For this study, the assessment will include events after the pandemic outbreak. The notion of 'traumatic events during pandemics' comprises Cov2-exposure and traumatic life events that occurred during the pandemics. 
COVID-19 Stress Scale $^{26}$ measures five groups of psychological problems related to the pandemic: (1) fear of contamination by COVID-19; (2) traumatic stress symptoms associated with direct or indirect exposure to the virus; (3) COVID-19-related compulsive checking and reassurance seeking; (4) xenophobic fears that foreigners are spreading COVID-19 and (5) worry about socioeconomic costs of COVID-19.

Perception of COVID-19 pandemic consequences on various aspects of life to explore strength and capacity for resumption of normal functioning during and after COVID-related stresses, included different areas of life-family relations, friendships, work/studies, physical activity, financial situation and internet use. We will ask if these areas of life were affected in positive, neutral, or negative directions (scale 1-5). The scale was created for this study by the authors.

\section{Predictor variables}

Basic personality traits. We will assess six basic personality traits (honesty, emotionality, extraversion, agreeableness, conscientiousness and openness) using a 60-item HEXACO inventory. ${ }^{27}$ We will also assess a disposition that is proposed by some authors as the separate personality trait, that is, disintegration, a proneness to psychotic-like experiences and behaviours, using a 20 -item Delta scale. ${ }^{28}$

\section{Mediator variables}

Loneliness will be assessed using a 3-item scale. ${ }^{29}$

Conspiracy about the origin of the COVID-19 virus will be measured using seven items (six of them reflecting the most popular conspiracies related to COVID-19 and one reverse keyed item articulating the official version of the COVID-19 origin).$^{30} 31$

Religious beliefs will be measured by one single question to find how participants appraise themselves.

\section{Moderator variables}

SF-municipality level contextual variables will be assessed (such as Gross Domestic Product (GDP), level of urbanicity, various demographic characteristics, level of employment, health service availability, numbers of those affected by COVID-19) ${ }^{32}$

\section{Data analysis plan}

Concerning the first objective, descriptive statistics will be reported for $\mathrm{MH}$ disorders and bivariate correlations will be used to present their relations with sociodemographic variables (such as gender, age, SES), Cov2-infection and somatic status. We also plan to investigate how QoL is associated with $\mathrm{MH}$ status.

Regarding the second objective, the primary dependent variables will be $\mathrm{MH}$ conditions (diagnostic groups and severity of most frequent MH conditions) and QoL. Predictors at level 1 will be traumatic events during pandemics, sociodemographic variables, personality traits and somatic status. Predictors at level 2 will be variables characterising municipalities where our respondents live. The hierarchical structure of the collected data (level 1-individual and level 2-SF) will be analysed using MRCM. ${ }^{33}$ This enables exploration of the moderation effects of SF on the relationships between individual factors (predictors and outcome measures).

Concerning the third objective, structural equation models will be used to investigate the mediator role of prepandemic indices of $\mathrm{MH}$ problems, loneliness, conspiracy and religious beliefs in the relationships between personality and sociodemographic variables on the one hand, and perception of pandemic consequences and distress on the other.

\section{Patient and public involvement}

No patients will be involved in the study. No formal public advisory committee was set up and there was no public involvement in the design and planning of the study. However, our participants will be informed that they can follow everything related to our study via our official website.

\section{ETHICS AND DISSEMINATION}

Ethical Committees of the Faculty of Medicine (1322VII/31) and Faculty of Philosophy in Belgrade (02-33/273) and Faculty of Philosophy in Novi Sad approved the protocol (05-27, br.893/1). Only respondents able to provide informed consent will participate in the study. Respondents can withdraw their consent and withdraw from the study. Respondents will be debriefed. Respondents who have elevated scores on the suicidal ideation subscale will be given a leaflet with national emergency help (SOS) phone lines, advising participants to contact their general practitioner/ nearest healthcare institution. Collected data will be completely anonymous. The anonymous dataset will be uploaded to a repository following all good scientific practices. RAs will be supervised throughout the data collection procedure.

Findings will be disseminated worldwide. A research report based on the study results will be submitted to peer-reviewed journals to be considered for publication. Results will be made available to funders, researchers, policy-makers, interested laypeople, through reports placed on the website www.cov2soul.rs, and advertised through social media.

\section{DISCUSSION}

The project will enable the assessment of $\mathrm{MH}$ disorder prevalence in a nationally representative Serbian sample. Moreover, this study was designed to investigate psychological and SFs serving as mechanisms for worsening MH. MH disorders will be assessed in person, using the stateof-the-art diagnostic tool by professionals familiar with the local context, comprehensively trained, and closely supervised throughout the data-collection process. This will enable comparison to other epidemiological studies using similar methodology and will contribute to the 
international evidence base on the epidemiology of psychiatric disorders following the COVID-19 pandemic.

Furthermore, the sampling procedure is designed to guarantee the representativeness of the sample for the general population of Serbia. Moreover, the study design (the hierarchical data organisation) enables thorough exploration of a unique combination of individual factors (ie, level 1 variables) and SF (at aggregated, municipal level, ie, level 2 variables) to understand the MH status of the general population, Cov2-distress (including COVID-19 health-related anxiety) and QoL.

This will be the first epidemiological study to provide nationally representative estimates for the prevalence of MH disorders for community-dwelling adults in Serbia, contributing to the global knowledge on the prevalence of mental disorders for the otherwise under-researched region of Europe. Although the comparison with data collected by Priebe et $a l^{34}$ - using a similar diagnostic interview on the Serbian population-is planned, the conclusions will be limited given the differences in the sampling procedures (participants in this research were identified in regions that had been directly exposed to war activities).

We also acknowledge an exceedingly small possibility that sampling strategy might underestimate the true prevalence of MH conditions in the population since institutionalised individuals will not be included. However, there will be no available information about the nonresponders, and we will not be able to conclude about this group of people, which could influence our results. Also, individuals who have been infected with COVID-19 and have persistent objectively measurable cognitive deficits, ${ }^{35}$ will remain understudied by the present research due to time and financial restraints. Finally, due to a cross-sectional design, it is not possible to unequivocally establish temporal precedence of the variables. That is, it could be that personality measurement or reported Cov2exposure is influenced by the pandemic-related changes in the MH status.

Long-term policies are required to meet the MH needs of populations affected by prolonged pandemic conditions and they should target groups with higher risks, which must be identified. In particular, limited access to $\mathrm{MH}$ care due to unavailability of MH services could negatively impact adequate diagnostic and therapy. ${ }^{1}$ This study will be able to provide clinically relevant information about the unmet needs of the population. Data from this study will be informative for public health institutions whose main areas of activity are analysis, planning and organisation of healthcare, health promotion, control and disease prevention, and QoL measurements. The public health sector will be provided with valuable information for further planning of prevention and implementation activities. Finally, the scientific community will benefit from the wide dissemination of the previously unavailable national data and with opportunities to include obtained data in forthcoming meta-analytical studies at European and other international levels.
Acknowledgements The authors would like to thank professor Stefan Priebe for thoughtful comments on the design of the study and to Kevin Saulnier for proofreading the manuscript.

Contributors NPM, GK and LBL designed the study, drafted the protocol and equally contributed to writing. OT and GK designed the sample. NPM, LBL, GK, LM, ZT and JT were involved with the creation of the test battery. NPM, LM, MPM and OV designed and conducted RA training and supervision procedures. All authors provided critical feedback on the protocol.

Funding This work was supported by the Science Fund of the Republic of Serbia, grant number \#7528289. The special research program on COVID-19 is financed by a World Bank loan through Serbia Accelerating Innovation and Entrepreneurship Project-SAIGE.

Competing interests None declared.

Patient and public involvement Patients and/or the public were not involved in the design, or conduct, or reporting, or dissemination plans of this research. Refer to the Methods section for further details.

Patient consent for publication Not applicable.

Provenance and peer review Not commissioned; externally peer reviewed.

Open access This is an open access article distributed in accordance with the Creative Commons Attribution Non Commercial (CC BY-NC 4.0) license, which permits others to distribute, remix, adapt, build upon this work non-commercially, and license their derivative works on different terms, provided the original work is properly cited, appropriate credit is given, any changes made indicated, and the use is non-commercial. See: http://creativecommons.org/licenses/by-nc/4.0/.

ORCID iD

Goran Knezevic http://orcid.org/0000-0001-8951-3774

\section{REFERENCES}

1 Holmes EA, O'Connor RC, Perry VH, et al. Multidisciplinary research priorities for the COVID-19 pandemic: a call for action for mental health science. Lancet Psychiatry 2020;7:547-60.

2 Mak IWC, Chu CM, Pan PC, et al. Long-Term psychiatric morbidities among SARS survivors. Gen Hosp Psychiatry 2009;31:318-26.

3 Lecic-Tosevski D, Vukovic O, Maric PB. The SAGE handbook of personality and individual differences. London: SAGE Publications Ltd, 2018: 90-114.

4 van Agteren J, Bartholomaeus J, Fassnacht DB, et al. Using Internetbased psychological measurement to capture the deteriorating community mental health profile during COVID-19: observational study. JMIR Ment Health 2020;7:e20696.

5 Brooks SK, Webster RK, Smith LE, et al. The psychological impact of quarantine and how to reduce it: rapid review of the evidence. Lancet 2020;395:912-20.

6 Galletly C. Psychiatry in the COVID-19 era. Aust N Z J Psychiatry 2020;54:447-8.

7 Winkler P, Formanek T, Mlada K, et al. Increase in prevalence of current mental disorders in the context of COVID-19: analysis of repeated nationwide cross-sectional surveys. Epidemiol Psychiatr Sci 2020;29:e173.

8 Pierce M, Hope $\mathrm{H}$, Ford T, et al. Mental health before and during the COVID-19 pandemic: a longitudinal probability sample survey of the UK population. Lancet Psychiatry 2020;7:883-92.

9 McCracken LM, Badinlou F, Buhrman M, et al. Psychological impact of COVID-19 in the Swedish population: depression, anxiety, and insomnia and their associations to risk and vulnerability factors. Eur Psychiatry 2020;63:e81.

10 Fiorillo A, Sampogna G, Giallonardo V, et al. Effects of the lockdown on the mental health of the general population during the COVID-19 pandemic in Italy: results from the comet collaborative network. Eur Psychiatry 2020;63:e87.

11 Patten SB, Kutcher S, Streiner D. Population mental health and COVID-19: why do we know so little? Can J Psychiatry 2021;19:7067437211010523.

12 Nezlek JB. Multilevel random coefficient analyses of Event- and Interval-Contingent data in social and personality psychology research. Pers Soc Psychol Bull 2001;27:771-85.

13 Stewart DE, Appelbaum PS. COVID-19 and psychiatrists' responsibilities: a WPA position paper. World Psychiatry 2020;19:406.

14 Fiorillo A, Gorwood P. The consequences of the COVID-19 pandemic on mental health and implications for clinical practice. Eur Psychiatry 2020;63:e32. 
15 Scherbaum CA, Ferreter JM. Estimating statistical power and required sample sizes for organizational research using multilevel modeling. Organ Res Methods 2009;12:347-67.

16 Hox J. Multilevel modeling: When and why. In: Classification, data analysis, and data highways. Berlin, Heidelberg: Springer, 1998: 147-54.

17 Faul F, Erdfelder E, Buchner A, et al. Statistical power analyses using $\mathrm{G}^{\star}$ Power 3.1: tests for correlation and regression analyses. Behav Res Methods 2009;41:1149-60.

18 Sheehan DV, Lecrubier Y, Sheehan KH, et al. The Mini-International neuropsychiatric interview (M.I.N.I.): the development and validation of a structured diagnostic psychiatric interview for DSM-IV and ICD10. J Clin Psychiatry 1998;59 Suppl 20:22-33.

19 Adler NE, Boyce T, Chesney MA, et al. Socioeconomic status and health. The challenge of the gradient. Am Psychol 1994;49:15-24.

20 Park HY, Park WB, Lee SH, et al. Posttraumatic stress disorder and depression of survivors 12 months after the outbreak of Middle East respiratory syndrome in South Korea. BMC Public Health 2020;20:1-9.

21 Kroenke K, Spitzer RL, Williams JB. The PHQ-9: validity of a brief depression severity measure. J Gen Intern Med 2001;16:606-13.

22 Spitzer RL, Kroenke K, Williams JBW, et al. A brief measure for assessing generalized anxiety disorder: the GAD-7. Arch Intern Med 2006;166:1092-7.

23 Priebe S, Huxley P, Knight S, et al. Application and results of the Manchester short assessment of quality of life (Mansa). Int J Soc Psychiatry 1999;45:7-12.

24 Ware J, Kosinski M, Keller SD. A 12-Item short-form health survey: construction of scales and preliminary tests of reliability and validity. Med Care 1996;34:220-33.
25 Brugha T, Bebbington P, Tennant C, et al. The list of threatening experiences: a subset of 12 life event categories with considerable long-term contextual threat. Psychol Med 1985;15:189-94.

26 Taylor S, Landry CA, Paluszek MM, et al. Development and initial validation of the COVID stress scales. J Anxiety Disord 2020;72:102232.

27 Ashton MC, Lee K. The HEXACO-60: a short measure of the major dimensions of personality. J Pers Assess 2009;91:340-5.

28 Knezevic G, Savic D, Kutlesic V, et al. Disintegration: a reconceptualization of psychosis proneness as a personality trait separate from the big five. J Res Pers 2017;70:187-201.

29 Hughes ME, Waite LJ, Hawkley LC, et al. A short scale for measuring loneliness in large surveys: results from two population-based studies. Res Aging 2004;26:655-72.

30 Bieber F, Prelec T, Jovic D. The suspicious virus: Conspiracies and COVID19 in the Balkans. BiEPAG, 2021.

31 Mulukom van V, Pummerer LJ, Alper S. Antecedents and consequences of COVID-19 conspiracy beliefs: a rapid review of the evidence. Available: https://psyarxiv.com/u8yah/

32 Gavrilovic D. Statistical yearbook, 2019. Available: https://www.stat. gov.rs/en-us/publikacije/publication/?p=12102

33 Lazarević LB, Bjekić J, Živanović M, et al. Ambulatory assessment of language use: evidence on the temporal stability of electronically activated recorder and stream of consciousness data. Behav Res Methods 2020;52:1817-35.

34 Priebe S, Bogic M, Ajdukovic D, et al. Mental disorders following war in the Balkans: a study in 5 countries. Arch Gen Psychiatry 2010;67:518-28.

35 Hampshire A, Trender W, Chamberlain S. Cognitive deficits in people who have recovered from COVID-19 relative to controls: an $\mathrm{N}=$ 84,285 online study. MedRxiv 2020. 